\title{
Arbor
}

\section{Métodos de análisis aplicados al estudio de la Obra de Arte}

\section{Carmen Muro García}

Arbor CLXIV, 645 (Septiembre 1999), 43-64 pp.

La contribución de disciplinas científicas como la química, física, biología, entre otras, al estudio de las obras de arte ha abierto en los últimos tiempos un fecundo campo de investigación, de intercambio y de colaboración interdisciplinar con el objetivo de mejorar el conocimiento y la conservación de nuestro patrimonio.

En este artículo se expone con carácter general el papel que crecientemente desempeñan los métodos de análisis aplicados a las obras de arte, sus principales objetivos y funciones y una breve visión de algunas de las diferentes técnicas empleadas.

El doble concepto de conocimiento y conservación como objetivo final de la investigación aplicada a este campo nos abre paso a una gran diversidad de funciones concretas que estas técnicas procuran: el estudio de la obra de arte en su etapa original, de sus transformaciones, de su estado de conservación, su cronología, la constatación de su autoría; así como también otros objetivos relacionados con la optimización de las técnicas de intervención y las condiciones de conservación preventiva.

Se exponen los objetivos y funciones de algunas de las principales técnicas usadas. Todas ellas ponen a disposición del análisis de la obra de arte un conjunto de informaciones que complementan el tradicional acercamiento a este ámbito de nuestra cultura. 


\section{Carmen Muro García}

\section{La colaboración interdisciplinar en el estudio de la obra de arte. Antecedentes históricos.}

La incorporación de determinados estudios científicos al campo de conocimiento de las obras de arte abre un sugestivo panorama de colaboración con los tradicionales métodos de investigación documental y estilística, y nuevas perspectivas en la documentación, conservación y difusión de una parte sustancial de nuestro patrimonio.

En este artículo se expone una visión general sobre este área científica desde la perspectiva de trabajo del Departamento de Conservación y Restauración de un museo de arte contemporáneo, como es el Museo Nacional Reina Sofía (M.N.C.A.R.S). Por ello, los ejemplos explicativos de las distintas técnicas utilizadas están seleccionados entre obras de arte contemporáneo.

Sin embargo, los principios que sustentan y cualifican la aplicación de la actividad científica dedicada al conocimiento y tratamiento de las obras.de arte son de carácter general, tanto para la obra de arte contemporáneo como para la de otras épocas históricas. En coherencia con ello, el desarrollo del presente artículo presenta un carácter global en relación al análisis de las obras de arte, aunque se señalan las diferencias y especificidades que presentan los bienes procedentes de la cultura contemporánea.

Esta creciente complementariedad entre datos derivados del estudio histórico y documental, o bien basados en la percepción visual y estilística, con los resultados ofrecidos por ciencias como la química o la física abre un nuevo campo de estudio multidisciplinar que se consolida como un sistema de trabajo que no existió como tal en épocas anteriores, aunque no faltan antecedentes puntuales de aportación de éstas al conocimiento de la obra de arte.

Selim Augusti definía tres períodos cronológicos de colaboración creciente entre el conocimiento técnico-matérico y el arte: "Un primer período, que comienza en la antigüedad clásica (con Plinio, Vitrubio, etc.) y que dura siglos, en el cual sólo hay ensayos aislados, en el primer momento más empíricos que científicos basados en análisis químicos, aplicados a objetos de interés arqueológico; un segundo período, que data de la segunda mitad del siglo XIX hasta los primeros años del siglo XX, durante el cual el intercambio de información y colaboración entre hombres de ciencias y hombres de letras y de arte comienza a sentar las bases cada vez más sólidas, $y$ durante el cual el análisis químico viene a añadirse a los métodos y medios de naturaleza fisica; un tercer periodo, que data de comienzos del siglo XX, en particular a partir de 1930, durante el cual los métodos científicos son aplicados también al examen directo de obras de 
arte y la colaboración entre la ciencia y el arte se convierte en una situación habitual»" ${ }^{1}$.

En efecto, estas tres etapas marcan tres estadios diferentes de la continua evolución de la colaboración interdisciplinar en el campo del estudio y conservación de obras de arte.

Concordante cronológicamente con el comienzo de ese citado segundo período de incipiente colaboración de la ciencia en el mundo del arte, es la célebre cita de Louis Pasteur, de 1865, donde pronosticaba la fecundidad de la colaboración entre las ciencias exactas y el mundo del arte. El 6 de mayo de dicho año afirmaba a los alumnos de la escuela de Bellas Artes en París: "Existen circunstancias en las que veo con claridad la alianza posible y deseable entre la ciencia y el arte, en la que el químico y el físico pueden ocupar un sitio junto a ustedes proyectando luz sobre sü trabajo» ${ }^{2}$.

Pero es a partir de principios del siglo XX, cuando los principales museos e instituciones comienzan a considerar la importancia de esta colaboración e integran dentro de sus equipos técnicos, los referidos a ciencias como la química, física, biología o la geología.

Existía el antecedente del laboratorio de Berlín, creado en 1888 para el estudio de Bienes Culturales, pero es después de la Primera Guerra Mundial cuando se comienzan a crear con cierto carácter sistemático estos equipos en los grandes museos del mundo occidental. Así, en 1931 se creó el Laboratorio para la Investigación de los Museos de Francia (L.R.M.F.) que en principio creció sólo en función del Museo del Louvre, hasta que en 1968, dio servicio a todos los museos de Francia. En Inglaterra se fundaron los laboratorios de la National Gallery y del British Museum, en Alemania el Doernert Institut en Munich; en Estados Unidos los del Metropolitan Museum y el de Smithsonian Institution, entre otros.

En la actualidad es una práctica habitual la colaboración interdisciplinar entre científicos de distintos campos en casi todos los principales museos e instituciones públicas y las principales privadas dedicadas a la conservación del patrimonio y al estudio de obras de arte.

\section{Especiales características del análisis de la obra de arte contemporánea.}

Paralelamente a esta evolución histórica de los avances metodológicos y científicos en el campo de la investigación de la obra de arte, se ha 


\section{Carmen Muro García}

producido un cambio fundamental en la propia elaboración de la obra artística, en concreto la pictórica.

Antes de la revolución industrial y de la aparición en el mercado de la pinturas comerciales, el artista era un conocedor de sus productos y colores, ya que elaboraba sus propias pinturas y conocía los materiales que utilizaba. El artista buscaba así las formulaciones más adecuadas que respondían a sus necesidades y que le permitirían desarrollar su labor creativa.

En 1841 la empresa "Claus \& Fritz" de Amsterdam comienza a producir pintura industrial en tubos de estaño. Dicha empresa se mantiene hasta 1931 y procuró material a varias generaciones de pintores, tal como reconocen cuarenta y tres artistas en un documento firmado el 30 de noviembre de $1941^{3}$.

La fabricación y distribución de pinturas proporcionó a los artistas nuevas posibilidades a la hora de plasmar su obra, pero a su vez les alejó en muchos casos de esta faceta que era parte importante de su aprendizaje y formación y que les permitía conocer y manipular los productos base de su obra creativa. Por otra parte, se comenzó a experimentar con nuevas formulaciones, escasamente ensayadas en algunos casos, lo cual puede conducir a resultados poco satisfactorios.

Este agudo contraste entre las circunstancias y posibilidades de la creación artística antes y después de la aparición de las pinturas industriales queda muy atinadamente reflejado por Dusan Stulik: "Antes de la revolución industrial y de la aparición de las pinturas fabricadas, el pintor no era sólo un artista, sino también un artesano de los colores. Mezclando los pigmentos con determinados aglutinantes (material que fija los pigmentos y pega la pintura al soporte), el pintor fabricaba sus propias pinturas y de este modo adquiría un conocimiento profundo de los materiales que utilizaba y de sus propiedades. Proliferaban asi fórmulas diferentes, ya que los artistas experimentaban con numerosos aglutinantes, buscando la combinación especial que daría a sus pinturas las propiedades ópticas y de textura.

Con la aparición en 1841 de la pintura de tubos y el desarrollo de la industria correspondiente, los pintores quedaron al margen del proceso de fabricación de las pinturas y perdieron la motivación para aprender los pormenores del oficio. Sin embargo, lograron una libertad considerable en el proceso creativo, ya que la pintura soluble les permitía salir de su estudio e idear nuevos estilos pictóricos" ${ }^{4}$.

Esta nueva naturaleza de la obra de arte contemporáneo matiza también la metodología de investigación, como es la del departamento de un museo destinado a obra de arte contemporáneo como es el Museo Nacional Reina Sofía, aunque básicamente los métodos instrumentales y los 
principios de investigación siguen siendo comunes a la obra de arte en general.

En efecto, la metodología de trabajo a seguir en el análisis de la obra de arte contemporáneo es básicamente similar a la que se lleva a cabo en las obras de épocas anteriores. Sin embargo, nos vamos a encontrar con las lógicas salvedades que se derivan de la diferente naturaleza de la obra a analizar. La diferencia básica radica en los materiales y técnicas empleadas. En la pintura contemporánea encontramos que hay una diversidad de técnicas y materiales, que incluyen desde los tradicionales a otros incorporados recientemente. Mientras que hay materiales tradicionales (aceites, huevo, resinas naturales, ceras,......) que se venían utilizando desde hace mucho tiempo, aparecen durante el siglo XX nuevos productos en las formulaciones de los artistas.

Para poner un ejemplo, si nos centramos en el tema de los pigmentos y cargas tradicionales, comprobamos que están constituidos en muchos casos por productos inorgánicos formados por óxidos, carbonatos, sulfuros, fosfatos y silicatos, entre otros. Durante este siglo se incorporan además nuevas formulaciones en los productos comercializados como materiales de artistas, de manera que se introduce un número considerable de pigmentos orgánicos e inorgánicos. Muy diferentes causas conducen a que durante la primera mitad de siglo aparezcan una serie de nuevos pigmentos inorgánicos. Entre otras causas, se encuentran algunas cuyo origen es ajeno al campo artístico, como por ejemplo el desarrollo de determinados materiales en época de guerra, o bien el resultado de la búsqueda de productos alternativos ante la toxicidad de determinados materiales, como el blanco de plomo.

Existe durante el siglo XX una amplia variedad de pigmentos disponibles para el artista contemporáneo. Resulta significativa la incorporación del blanco de titanio, dióxido de titanio, introducido en 1918, o bien los cadmopones, el azul de manganeso y una larga lista de compuestos.

Por otro lado, la bibliografía describe aproximadamente quinientos pigmentos orgánicos adecuados para el uso de los artistas que se han desarrollado en los últimos cincuenta años, de los cuales la mitad se han distribuido comercialmente.

Además, la investigación en química orgánica ha generado numerosos materiales sintéticos, que se han utilizado como barnices y aglutinantes. Entre ellos podemos citar las resinas alquídicas, acrílicas o vinílicas, así como otras pinturas en suspensión acuosa.

Algunos de estos nuevos aglutinantes fueron introducidos en el mercado americano a finales de los años cincuenta. Sin embargo, hizo falta esperar hasta la mitad de la siguiente década para que el uso de los aglutinantes acrílicos, por ejemplo, se extendiera en Europa. Actualmente se 


\section{Carmen Muro García}

estudia por diferentes grupos de investigadores el momento en que tuvo lugar la síntesis y la incorporación como materiales para artistas de diferentes productos empleados durante el siglo XX. Esta identificación puede aportar puntos de referencia cronológicos, que en su caso permitirán verificar la autenticidad de una obra o precisar la naturaleza de una intervención posterior al proceso de ejecución. De la misma manera, el conocimiento de estos materiales se convierte en premisa para resolver problemas de conservación. Se trata además de conocer su historia y el desarrollo de cada uno de ellos. Ello es debido a que su incorporación tuvo relación con diferentes razones, como pueden ser las propiedades ópticas, la resistencia a la luz, el brillo o el bajo coste, de manera que todos éstos son factores que condicionan su empleo.

En el apartado de las diferencias y similitudes de la obra contemporánea con respecto a la obra antigua, nos encontramos con los siguientes aspectos: una diferencia básica es que, al existir mayor posibilidad de empleo de materiales, el campo de experimentación es crecientemente más amplio y diversificado $\mathrm{y}$, como consecuencia, en algunos casos hay una cierta despreocupación en relación a la durabilidad de la obra; otro factor que nos diferencia -en cuanto al estudio de la obra- es que podemos encontrar materiales cuyo comportamiento no somos capaces de prever a largo plazo, ya que no se conoce claramente su evolución, a diferencia de la obra antigua cuya transformación a través del tiempo está más caracterizada.

Pero frente a este panorama general, también observamos ciertas ventajas cuando nos enfrentamos al estudio de la obra contemporánea, ya que contamos con la posibilidad de conocer mayor número de datos sobre el proceso de creación, bien a través del artista vivo, que puede colaborar y ofrecer información al respecto, o bien familiares o personas cercanas que pueden ofrecer un similar nivel de aproximación. Igualmente, al estar estas obras mucho más cercanas en el tiempo, hay una mayor y más variada documentación sobre ellas en cuanto a grabaciones, videos, escritos, etc. Todos estos datos, si existen, son puntos de referencia muy valiosos a la hora de abordar el análisis.

\section{Funciones de la actividad científica en relación a la conservación de obras de arte.}

La actividad científica, de investigación cubre, con respecto a las obras de arte, una serie de funciones que se desarrollan como parte de la misión de los museos y también de los organismos e instituciones -públicas o privadas-encargadas de la conservación del patrimonio. 
Los objetivos de la investigación científica sobre la conservación de obras de arte se centran en los siguientes campos:

\section{- El conocimiento de la obra.}

Se trata de un área de investigación básica, inexcusable para todas las demás aproximaciones a la conservación. Es necesario conocer el estado material de las obras de arte, su estructura, su técnica de ejecución, su composición material: soportes, aglutinantes, capa pictórica en su caso. El conocimiento en profundidad, desde la aportación de la ciencia, de las obras de arte constituye la base de actuación, cuya caracterización es absolutamente necesaria para poder establecer las ulteriores aproximaciones a su estado de conservación, a las posibles intervenciones o a las medidas de mantenimiento preventivo.

La obra que hoy conocemos y que percibimos como objeto de patrimonio, como bien cultural, es el resultado de un proceso cuyo origen es un acto de creación artística, pero que con posterioridad ha tenido una historia más o menos larga o compleja, habitualmente teñida de acontecimientos: traslados, intervenciones, daños.

$\mathrm{El}$ análisis de la obra de arte incluye esta dimensión temporal necesaria para su comprensión. Por tanto, se estudian los siguientes aspectos:

- La estructura material de la obra original.

- La influencia del tiempo en la evolución de los materiales.

- Las intervenciones ejecutadas sobre ella, tanto en épocas más antiguas, en muchas ocasiones barnices o repintes, como actuaciones más modernas de restauración, ya con criterios actuales de conservación del patrimonio.

- Los daños sufridos por la obra de arte. En ocasiones estos daños son excepcionales, debido a traslados, catástrofes naturales, agresiones. En otros casos son debidos a las inadecuadas condiciones de conservación.

- En ocasiones es objeto de la investigación incluso la existencia en el soporte de composiciones materiales, anteriores a la obra de creación que contemplamos en la actualidad.

De acuerdo con lo anteriormente expresado, en muchas de las obras el análisis se centra sobre todo, al menos desde el punto de vista general, en caracterizar la técnica material, composición y estructura de la obra original. Pero, efectivamente, también ha de tenerse muy en cuenta toda esta dimensión temporal de la obra, según los epígrafes anteriormente expuestos, ya que se trata de adquirir una visión global del bien cultural analizado.

Los objetivos de este conocimiento de la obra son muy diversos. No en vano la caracterización más profunda de esta obra nos proporciona la ba- 


\section{Carmen Muro García}

se de las demás investigaciones o intervenciones. Dichos objetivos comprenden un conjunto que exponemos a continuación:

- La difusión del conocimiento sobre el Bien Cultural. Este es un objetivo inmediato y básico de toda investigación: el aumento del saber, del conocimiento para su transmisión a través de publicaciones especializadas o divulgativas. Ampliar el campo del conocimiento humano y transmitirlo es un objeto en sí mismo de la investigación.

- Establecer las bases para ulteriores actividades de conservación. $\mathrm{Pa}$ ra esta actividad de conservación, que es una de las funciones básicas de las instituciones relacionadas con el patrimonio cultural, es conveniente, cuando no imprescindible, un afinado conocimiento de la realidad material de las obras de una determinada época o autor, y en concreto de la obra objeto de la intervención de conservación. El incremento paulatino y continuo de datos y autores, así como de los procesos ulteriores sufridos por las obras nos ofrecen información valiosísima para el conocimiento comparado, que sirva de base para una correcta estrategia de conservación.

- Contribución a la datación cronológica o autentificación son otros objetivos, en este caso más concretos, de la investigación básica sobre las obras de arte. La utilidad de estas dos funciones abarca campos muy variados en relación a la adquisición de obras por parte de los museos o instituciones de patrimonio, al comercio de obras de arte, a la actividad pericial.

\section{- El análisis del estado de conservación.}

Este es otro de los objetivos principales de la investigación científica que estamos exponiendo en relación a las obras de arte.

Los análisis van encaminados no sólo a la caracterización objetiva de la composición material de la obra, sino también a establecer y conocer con el mayor grado de profundidad posible el estado de conservación.

Las distintas técnicas empleadas en los análisis nos permiten aproximarnos al conocimiento de los procesos de deterioro del soporte y de la capa pictórica, en su caso, de los daños físicos que sufre la obra, de las alteraciones de origen químico o biológico, las pérdidas, así como también de la posible incidencia negativa de determinadas intervenciones realizadas en el pasado: traslados, restauraciones inadecuadas, deficientes condiciones ambientales.

Los resultados de esta investigación sobre el estado de conservación de la obra de arte y el subsiguiente diagnóstico deben constituir un punto de partida firme y objetivo -junto con el que ofrezca la investigación documental, histórica y estilística- para la estrategia de conservación, en el sentido que éste tenga que manifestarse tanto en el apartado de medidas de conservación preventiva como en el de intervención de restauración. 
- Apoyo y colaboración a la actividad de restauración.

Del análisis de las obras de arte y, en particular, de su estado de conservación derivan numerosas constataciones que pueden resultar útiles en relación a la actividad de restauración.

En efecto, todo el conocimiento aportado sobre cómo soportan el paso del tiempo los distintos tipos de materiales, de soportes, de aglutinantes, de pigmentos o barnices, así como de la técnica material empleada ofrecen datos en relación a los procesos de restauración.

Pero también, el análisis anteriormente expuesto de las restauraciones realizadas en otras épocas sobre las diferentes obras de arte constituye en sí mismo una relevante fuente de información sobre el funcionamiento a medio y largo plazo de determinadas técnicas empleadas y de los materiales utilizados. Bien es verdad que los materiales y sistemas de restauración evolucionan a considerable velocidad y lógicamente hay que dar por supuesta la obsolescencia de algunas de las restauraciones y técnicas empleadas en el pasado, pero el análisis comparativo entre ellas y su distinto comportamiento al paso del tiempo nos puede aportar muchas sugerencias en relación a los sistemas de restauración a utilizar en el presente.

Además de las aportaciones que el análisis de las obras puede suponer para las actividades de restauración, la misión de un laboratorio de análisis aplicados a la conservación tiene un campo muy amplio de trabajo en el examen de los distintos sistemas de restauración y de los materiales utilizados, tanto mediante el análisis directo de los materiales como la necesaria actividad de seguimiento de las obras ya restauradas. También hará referencia a los posibles efectos secundarios, así como al carácter de reversibilidad o irreversibilidad de los materiales utilizados. De esta actividad parte un conjunto de iniciativas para el conocimiento cada vez mas firme de las distintas técnicas utilizadas en el campo de la restauración y de su idoneidad y eficacia en cada caso en función del tipo de obra a restaurar y de los problemas diagnosticados.

- Contribución al establecimiento de medidas de conservación preventiva.

Cada vez se percibe con mayor nitidez la importancia de una adecuada conservación preventiva en la estrategia global de la protección y conservación de las obras de arte. La relación de los departamentos de conservación de los museos con las actividades y medidas de conservación preventiva desempeña, por tanto, cada vez un papel más desarrollado.

Las condiciones ambientales -humedad, temperatura, iluminación,...- que deben mantener las obras de arte precisa un profundo cono- 


\section{Carmen Muro García}

cimiento de cada una de ellas en función del tipo de material y de su estado de conservación, en el que encuentra una considerable responsabilidad el departamento de restauración. Lo mismo ocurre con todo lo relativo a las cada vez mas frecuentes exposiciones, con todo lo que ello lleva consigo en relación a traslados, embalaje, cambio en las condiciones ambientales, almacenamiento, transporte, actividades más o menos cruentas cuyas consecuencias sobre los bienes culturales es preciso minimizar.

\section{Métodos de análisis.}

En este apartado se expone la metodología de investigación basada en las técnicas analíticas y en su aplicación a las obras de arte, dentro de las funciones habituales del laboratorio de química de un museo.

Para conseguir un acercamiento a la composición material de una obra de arte disponemos básicamente de dos fuentes complementarias de información. Por un lado, contamos con una aproximación de carácter global, que se basa en la utilización de la radiación electromagnética en un amplio rango de longitudes de onda, y que va a permitir generar una imagen del objeto visible a simple vista. Nos referimos, entre otras, en concreto a la información que proporcionan técnicas tales como la reflectografía infrarroja o la radiografía. Ambas son técnicas de análisis no destructivas aplicadas a las obras de arte. Los exámenes de este tipo pueden incluirse en un grupo de análisis globales.

Estos exámenes son absolutamente necesarios y deben preceder siempre al uso de una segunda fuente de información, donde la composición material de áreas, que aunque sean pequeñas han de ser representativas, va a ser determinada por métodos analíticos. Esto es lo que podemos llamar análisis puntual, y es el que aparece más comúnmente vinculado a la práctica habitual del laboratorio de química , que incluye técnicas como microscopía óptica, espectroscopía infrarroja, cromatografía de gases o microscopía electrónica, entre otras, cuya aplicación se expondrá más adelante.

El continuo desarrollo de las técnicas instrumentales permite un progresivo acercamiento, una nueva percepción, en cuanto a la composición y el comportamiento de los materiales.

Existe una gran diversidad de métodos de investigación aplicables al conocimiento de obras de arte. Muchos de ellos derivan de técnicas empleadas con carácter general en el estudio de materiales y que han sido notablemente desarrolladas. La investigación científica sobre las obras de arte es un campo de aplicación de muchas de estas técnicas. 
Encontramos una gran variedad de sistemas de caracterización de las técnicas y materiales de la obra de arte que se refieren a las diferentes partes del conjunto de la obra como pueden ser el soporte, los materiales de capa pictórica u otros materiales incorporados con posterioridad o bien productos de alteración formados con el paso del tiempo. Todo ello sin olvidar la evaluación de las nuevas formulaciones susceptibles de ser incorporados como productos aplicables en la restauración, analizando su idoneidad y eficacia, como dijimos anteriormente.

Si repasamos brevemente el esquema general de algunos de los métodos instrumentales analíticos utilizados en el campo de la conservación (Fig.1), vemos que comprenden desde los métodos instrumentales clásicos hasta otros incorporados más recientemente, como son las técnicas láser LIBS o LIF, o técnicas basadas en la emisión de rayos $\mathrm{X}$ o gamma inducidos por partículas, como las técnicas PIXE o PIGE.

A la vista de este esquema es importante recalcar el carácter no destructivo de algunas de las microtécnicas y técnicas de análisis de superficie, o bien, el requerimiento de pequeñísimas cantidades de material, lo

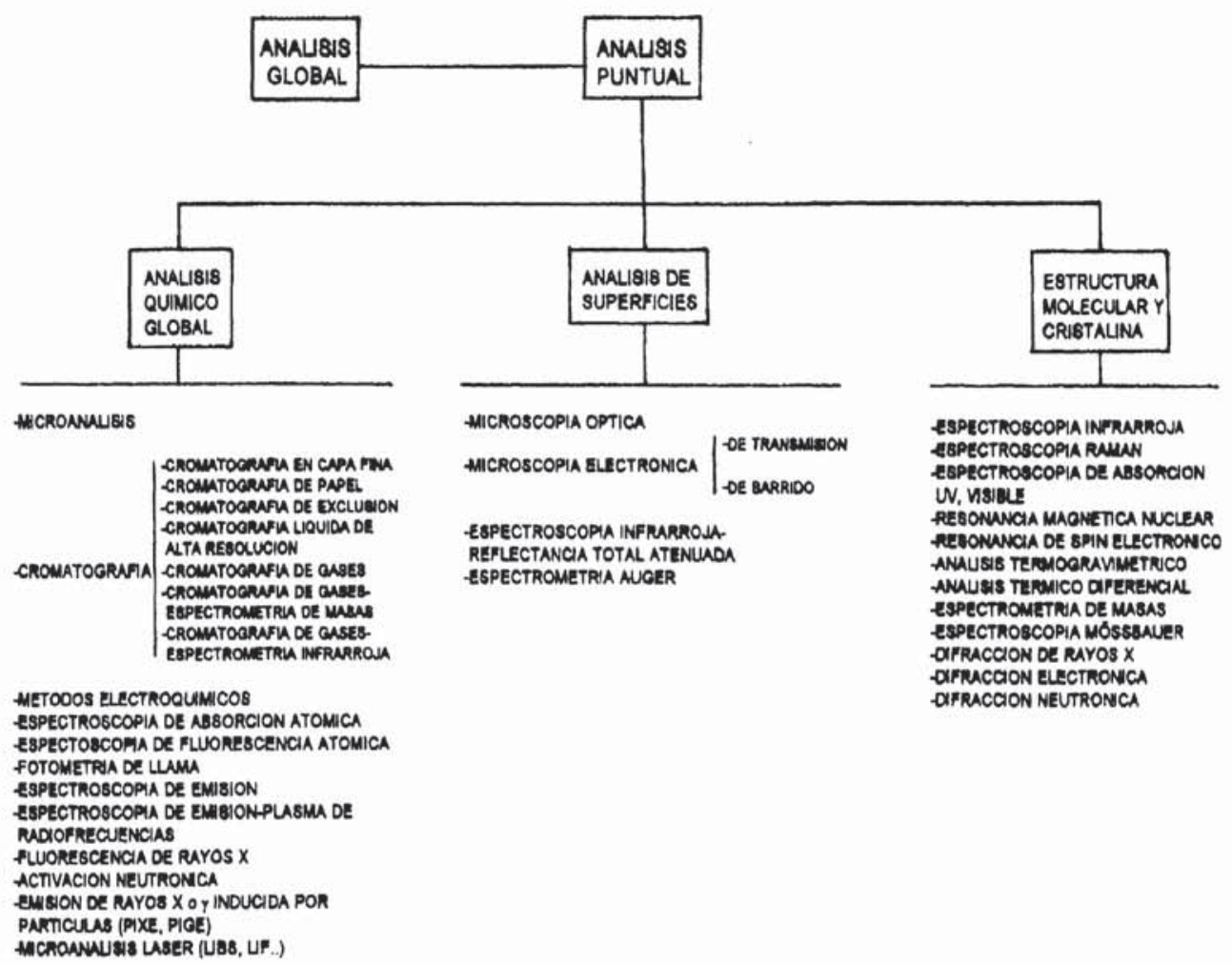

Figura 1. Esquema general de algunos de los métodos instrumentales analíticos utilizados en el campo de la conservación. 
cual constituye una ventaja o, mejor dicho, un requisito a la hora de analizar objetos de arte, que es el objeto de nuestra actividad.

Apoyándonos en las técnicas de análisis global, como hemos señalado inicialmente y según los objetivos del análisis, elegimos los métodos a emplear. Sobre este esquema de técnicas en general aplicables en el campo de la conservación vamos a destacar las más habituales, que abarcan desde microscopía óptica a técnicas cromatográficas. Vamos a exponer brevemente estos procedimientos.

El microscopio óptico encuentra muchas aplicaciones en conservación, para el estudio de muy diferentes tipos de materiales como fibras textiles y de papel, metales, piedra, vidrio, madera. Se trata de una técnica básica que nos da información sobre la morfología de partículas y estructuras, bien sobre capas de pintura o sobre soportes. Por tanto, contribuye al examen de las superficies de pintura y de las secciones transversales a la hora de identificar pigmentos, reconocer tratamientos superficiales y estudiar la estructura en capas. Se puede, mediante esta técnica, desvelar adiciones posteriores en una obra o bien conocer el estado material en cuanto a la adhesión o cohesión de los diferentes estratos en una superposición de capas de pintura.

Este primer examen se completa con ensayos microquímicos con reactivos específicos, que nos ponen de manifiesto la existencia de determinados materiales en la zona analizada, tanto referidos a pigmentos como a aglutinantes. Existe una amplia gama de ensayos microquímicos de tinción específicos para proteínas o aceites, por ejemplo, que nos ayudan a visualizar la presencia o ausencia de los mismos en la zona estudiada. También se emplean secuencias de ensayos microquímicos a la hora de abordar el estudio de fibras textiles y de papel.

Para permitir la manipulación de las pequeñísimas muestras de las que podemos disponer, en muchas ocasiones se recurre a la inclusión de las mismas en una resina apropiada que permita el corte y el pulido hasta obtener una superficie que presente la sección transversal y que nos va a poner en disposición de realizar las sucesivas pruebas sobre la misma muestra.

La superficie es examinada con luz reflejada o transmitida dependiendo de la naturaleza de la muestra a estudiar, y por tanto del examen específico que sea preciso.

Vamos a ver algunos ejemplos referidos, en primer lugar, a la identificación de las fibras de soportes. Esta se realiza mediante el examen microscópico de muestras tomadas en ambos sentidos de una tela, urdimbre y trama, observadas en sentido longitudinal. Así, en el cuadro de Benjamín Palencia, titulado "Composición" (1935), se ha identificado algodón como componente de la trama. Esto se pone de manifiesto por el análisis 
Métodos de análisis aplicados al estudio de la Obra de Arte

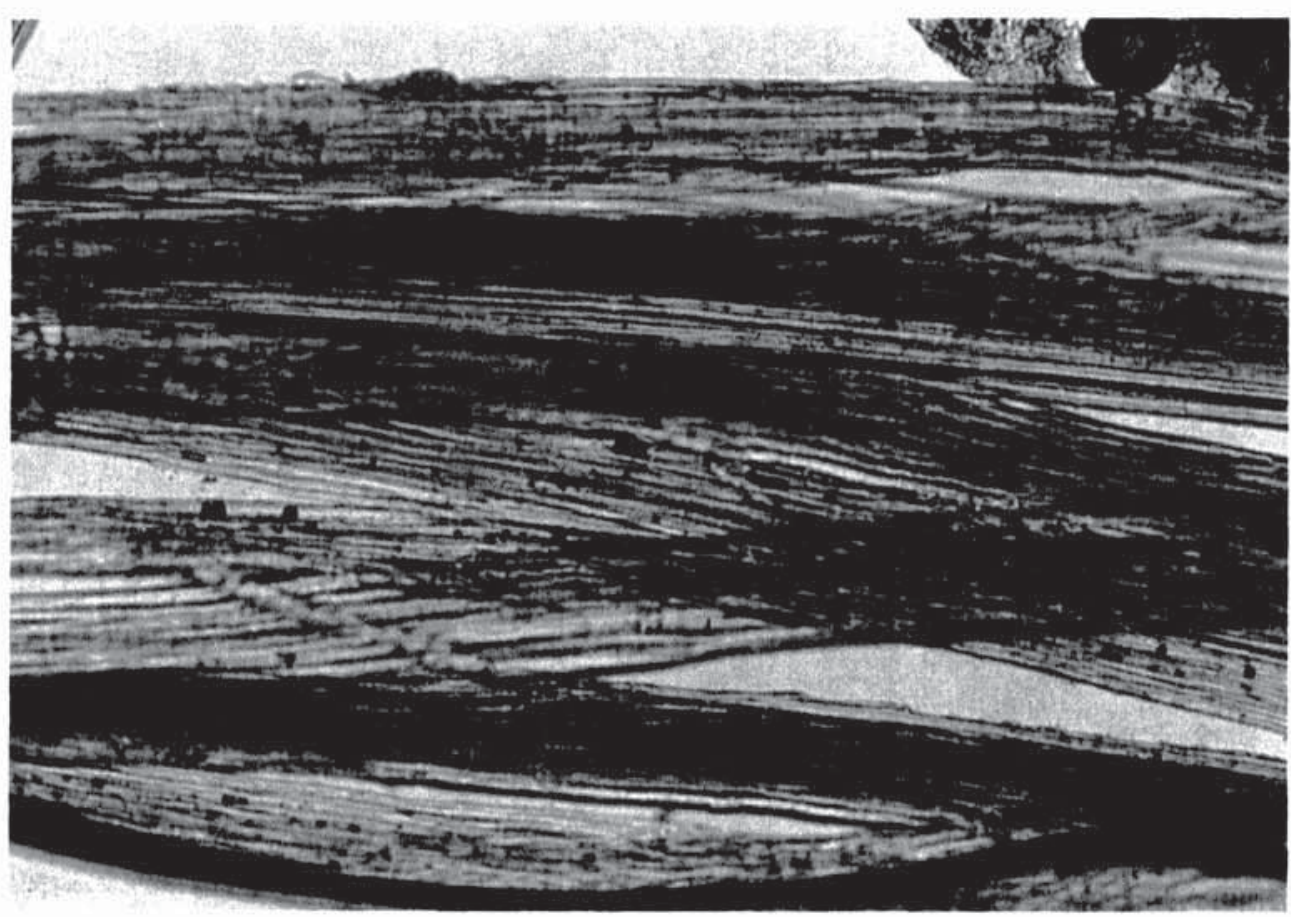

Figura 2. Yute, vista longitudinal. Ensayo con floroglucina en medio clorhidrico.

morfológico característico de esta fibra, mientras que se ha identificado en urdimbre yute, tal como se aprecia en la (Fig.2). En efecto, el color magenta característico, que está obtenido al realizar el ensayo con floroglucina en medio clorhídrico, pone de manifiesto el alto contenido de lignina de esta fibra. Esto, a su vez, queda corroborado en el corte transversal por los manojos de las fibras elementales poligonales y el lumen circular o elíptico que se aprecia en un corte transversal de estas mismas fibras (Fig.3). El hecho de la diferente composición puede ser un dato sobre el posible comportamiento diferencial entre urdimbre y trama en esta tela, por ejemplo frente a una ulterior intervención.

Pero además este examen por microscopía óptica contribuye al conocimiento de las superficies de pintura y de las secciones transversales a la hora de identificar los pigmentos y la estructura en capas. Igualmente, se pueden desvelar adiciones posteriores o el estado material en cuanto a adhesión y cohesión de los estratos. Este examen se completa con ensayos microquímicos con reactivos específicos, que ponen de manifiesto la presencia de determinados materiales en las muestras analizadas.

En este sentido y a modo de ejemplo nos vamos a referir a un cuadro de Wifredo Lam. Este pintor cubano es uno de los más prestigiosos pin- 


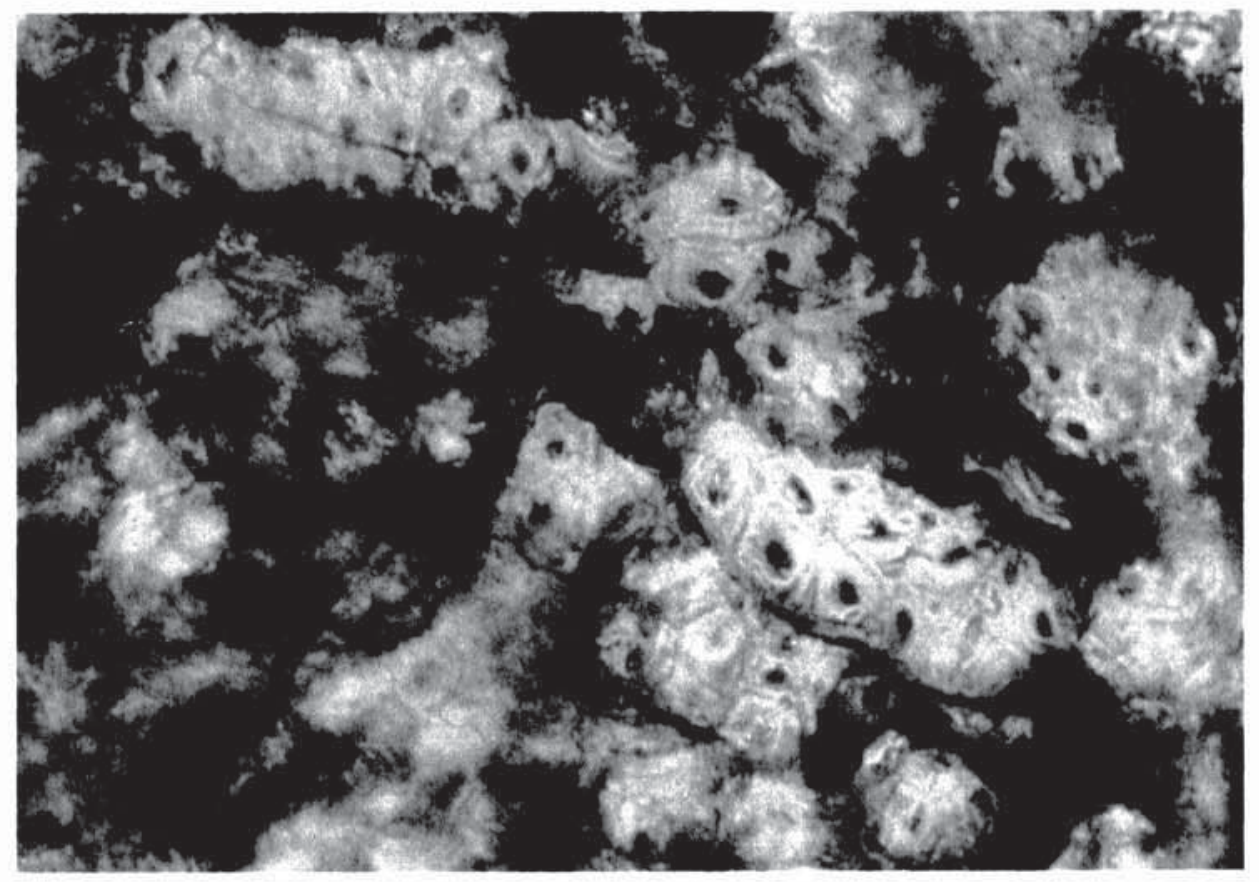

Figura 3. Yute. Sección transversal de los manojos de las fibras elementales.

tores americanos de este siglo; se formó y vivió quince años en España. El cuadro analizado se titula "Paisaje» y está firmado y fechado en 1932. La imagen de la fluorescencia visible obtenida a partir de la radiación ultravioleta, nos permite detectar las zonas con adiciones y aquellas otras inalteradas, paso necesario y previo a cualquier otro análisis puntual. De esta forma se plantea el esquema de toma de muestras, en aquellas zonas más representativas y que tengan menor incidencia en la superficie.

Ahora bien, el examen por microscopía óptica de muestras incluidas en una resina nos permite, por ejemplo, ver esta muestra azul del cielo (Fig.4), cuya sección transversal revela que la capa más externa contiene azul ultramar. En esta misma muestra se realizó un ensayo microquímico con el reactivo Negro Sudán B, cuyo resultado positivo fue indicativo de la presencia de una técnica de ejecución al óleo.

En el cuadro de Antonio López, titulado "El muchacho del tirador" de 1953 , se observa una sección transversal de una muestra verde mate, que contiene óxido de cromo anhidro $-\mathrm{Cr}_{2} \mathrm{O}_{3}-$ (Fig.5). Si centramos la atención en la preparación, observamos que da positiva la tinción con el reactivo Fuchsina neutra (Fig.6), que nos indica la existencia de proteína en la zona de preparación. 


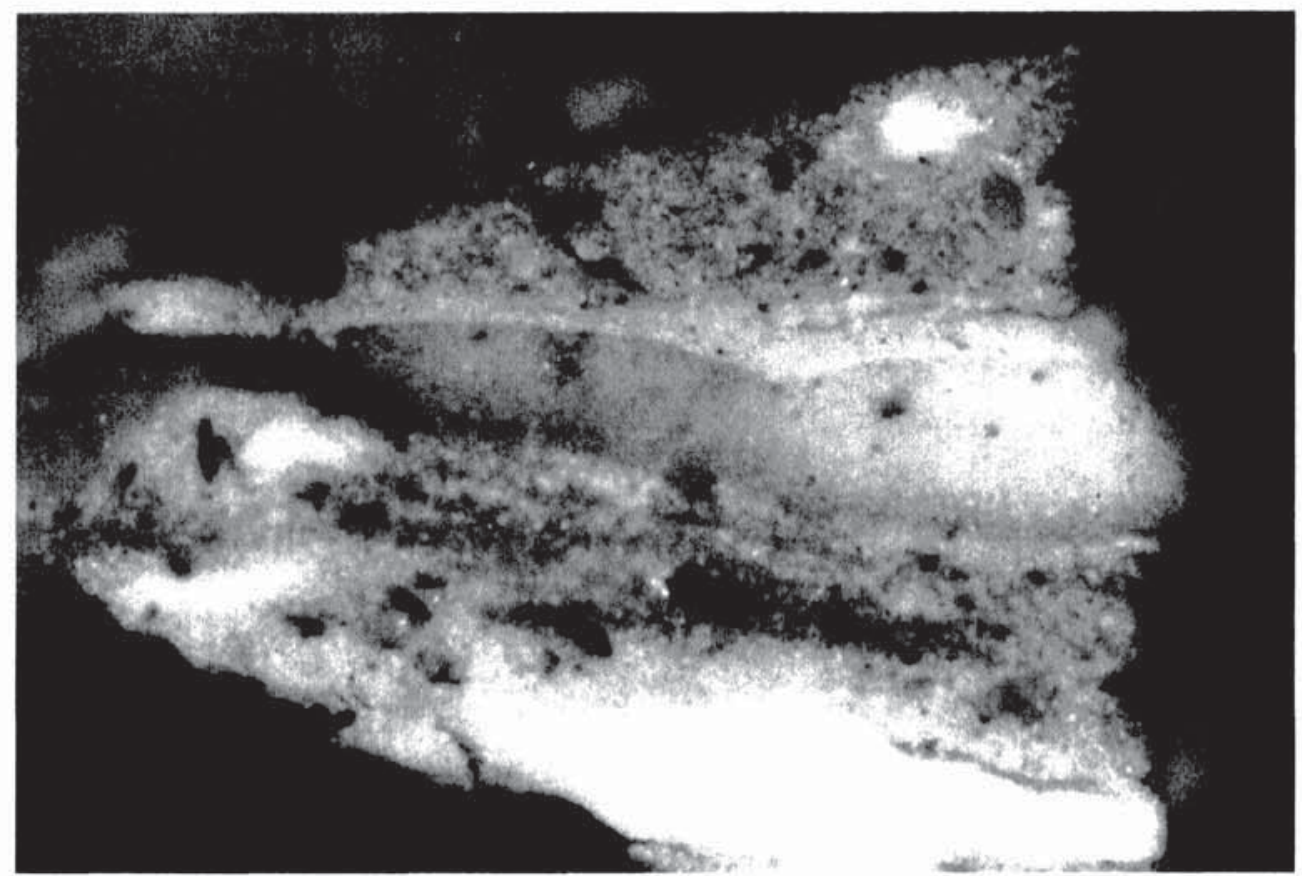

Figura 4. Superposición de capas de una muestra del azul del cielo de la obra „Paisaje» de Wifredo Lam.

Como veíamos en el esquema inicial, asimismo utilizamos métodos que nos proporcionan información sobre la estructura molecular de los componentes presentes en las muestras. En este sentido, las técnicas espectroscópicas de absorción son adecuadas para la identificación de determinados materiales, tanto orgánicos como inorgánicos.

Con la técnica de espectrometría infrarroja es posible examinar los diferentes tipos de vibraciones de enlace químico que tienen lugar entre los átomos de una molécula, mediante la medida de las energías características asociadas con estos movimientos. Cuando las vibraciones moleculares dan lugar a un cambio en el momento dipolar de enlace, como consecuencia del cambio de la distribución electrónica en el enlace, es posible estimular transiciones entre diferentes niveles de energía por interacción de la radiación electromagnética de la frecuencia apropiada.

En concreto, el examen por espectroscopía infrarroja nos facilita la medida de esta absorción infrarroja realizada por la muestra. Por tanto, el espectro infrarrojo de un compuesto es esencialmente la superposición de las bandas de absorción características de los grupos funcionales específicos, como si se tratara de una huella del compuesto, aunque las interacciones débiles con los átomos circundantes le confieren un sello de individualidad al espectro. Por ejemplo, no va a ser lo mismo que estemos frente a un compuesto aromático mono o disustituido, en posición orto-, 


\section{Carmen Muro García}

58

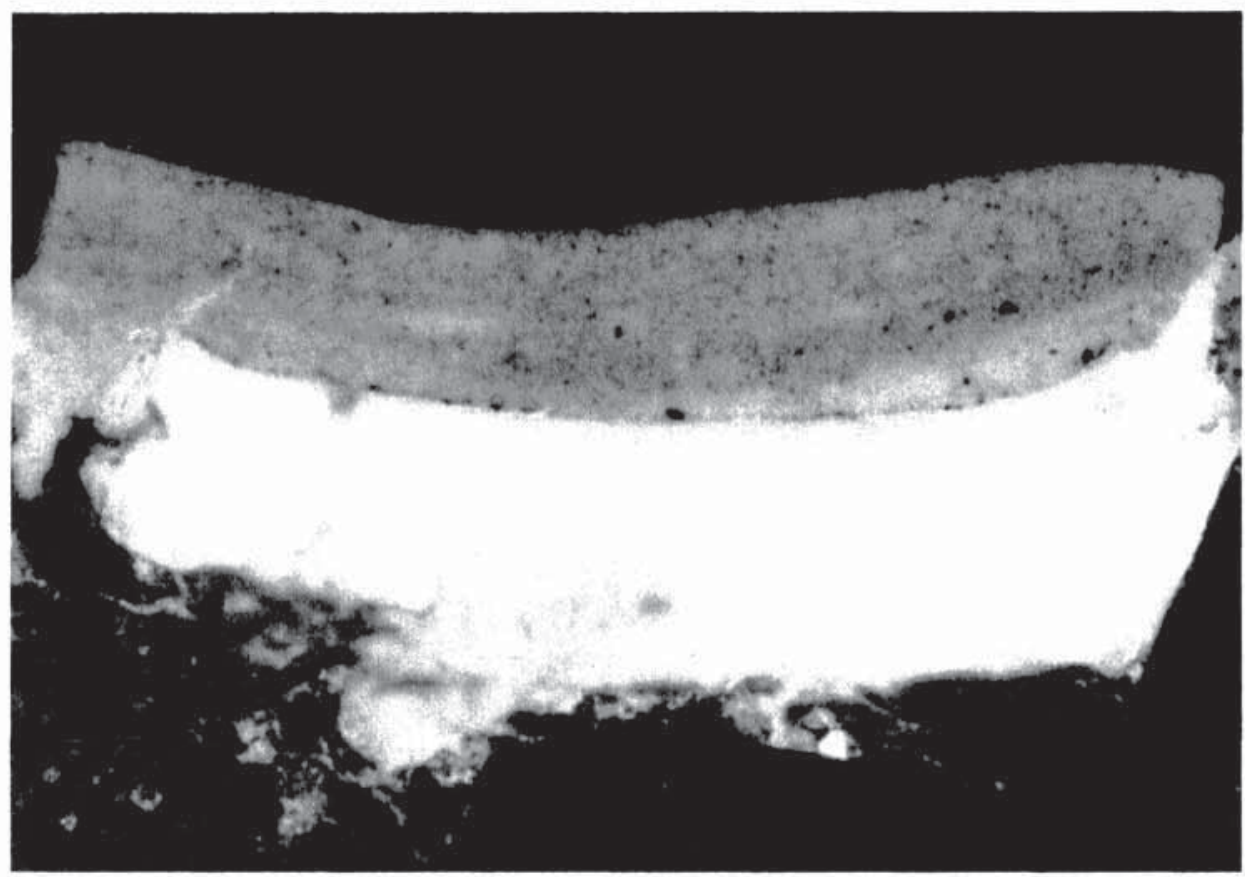

Figura 5. Microfotografía de una muestra verde procedente de la zona de la fachada en "El muchacho del tirador de Antonio López, cuya capa pictórica contiene verde de óxido de cromo opaco.

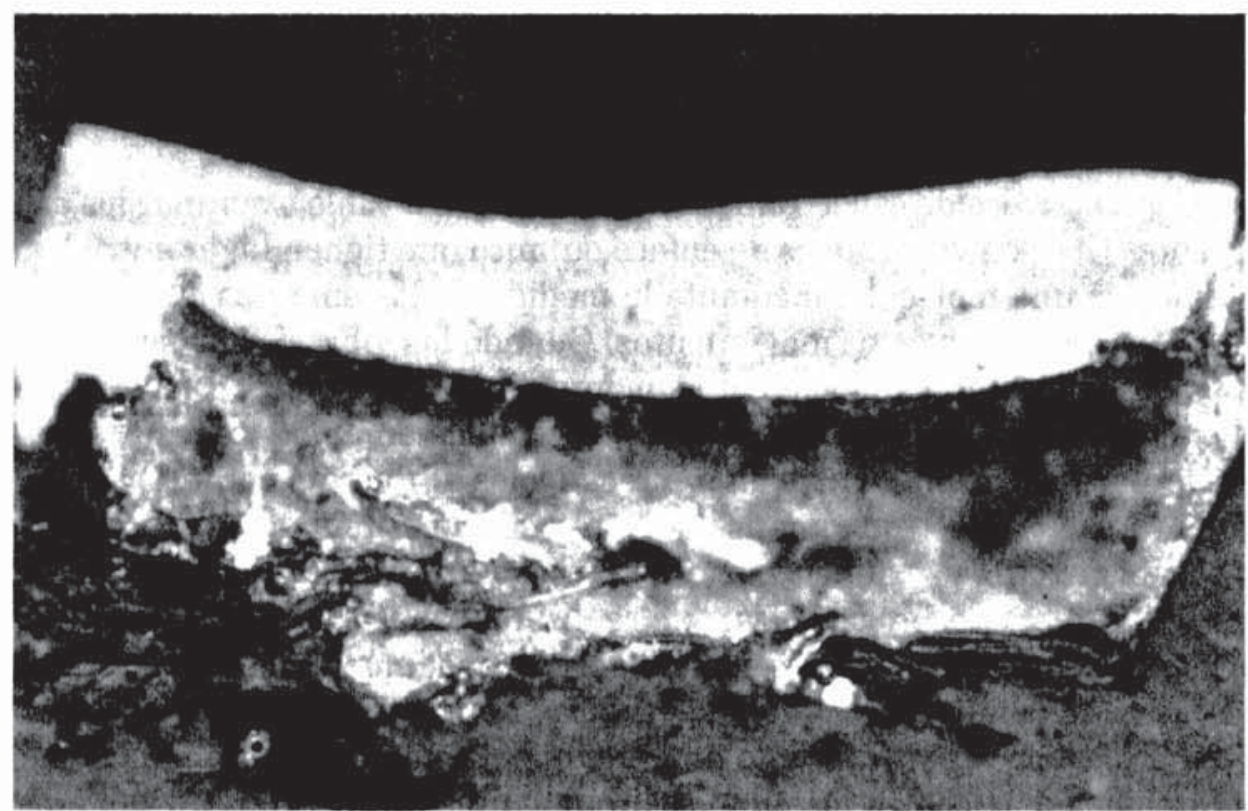

Figura 6. Tinción con el reactivo Fuchsina de la muestra de la Figura 5. 
meta- o para-, que esté condensado o no, o bien que nos encontremos ante un grupo ester procedente de un acetato o de un acrilato.

Cuando se interpreta un espectro es posible establecer qué grupos funcionales se encuentran presentes mientras qué otros están ausentes. A veces, con sólo estos datos las diferentes posibilidades de identidad son tales que se puede hacer una interpretación que permita la identificación. En otras ocasiones, la interpretación del espectro infrarrojo en función de las frecuencias características no basta para identificar el problema total, pero permite distinguir qué tipo de compuesto se analiza. Hemos hablado antes de la superposición de bandas de absorción de los grupos funcionales presentes, de manera que en el caso de mezclas, que es lo habitual, la complejidad aumenta considerablemente debido a la superposición de diferentes componentes.

Existe la ventaja adicional de que la técnica de microespectroscopía infrarroja por transformada de Fourier permite analizar muestras extremadamente pequeñas. Así, por ejemplo, vamos a poder realizar el análisis de los diferentes componentes presentes en las capas en una sección transversal de pintura, o bien, productos de deterioro surgidos como resultado del envejecimiento de algunos materiales y que pueden dar importante información sobre los problemas de degradación de los mismos.

La información así obtenida nos dispone a plantear una identificación del material presente. En consecuencia se ha aplicado fundamentalmente al examen de aglutinantes, barnices, adhesivos, pigmentos, productos de alteración, resinas, ceras y un amplio rango de productos naturales y sintéticos.

En el cuadro anteriormente citado «El muchacho del tirador» de Antonio López encontramos en la preparación $\mathrm{BaSO}_{4}$, como vemos en el espectro de la (Fig.7), además de carbonato, en concreto $\mathrm{CaCO}_{3}$ (bandas a 1428,876 y $713 \mathrm{~cm}^{-1}$ ) y cola, tal como anunciaba el ensayo de tinción de proteínas que habíamos visto antes.

Esta técnica puede, igualmente, en el caso de la identificación de materiales añadidos, aportar una información muy útil para una intervención posterior.

Esta técnica puede ser complementada por otras, como la cromatografía de gases, que veíamos inicialmente en el esquema general. Puesto que los aglutinantes y barnices son generalmente mezclas, una más precisa identificación requiere la separación y cuantificación de los componentes químicos individuales. La cromatografía de gases es un método idóneo para este objetivo. Es muy sensible y, por tanto, es la técnica a elegir para la separación de componentes que están presentes en muchas muestras a la hora de abordar el estudio de aglutinantes y recubrimientos en general. Se utiliza para el análisis de aceites, resinas, ceras y gomas, entre otros. 


\section{Carmen Muro García}

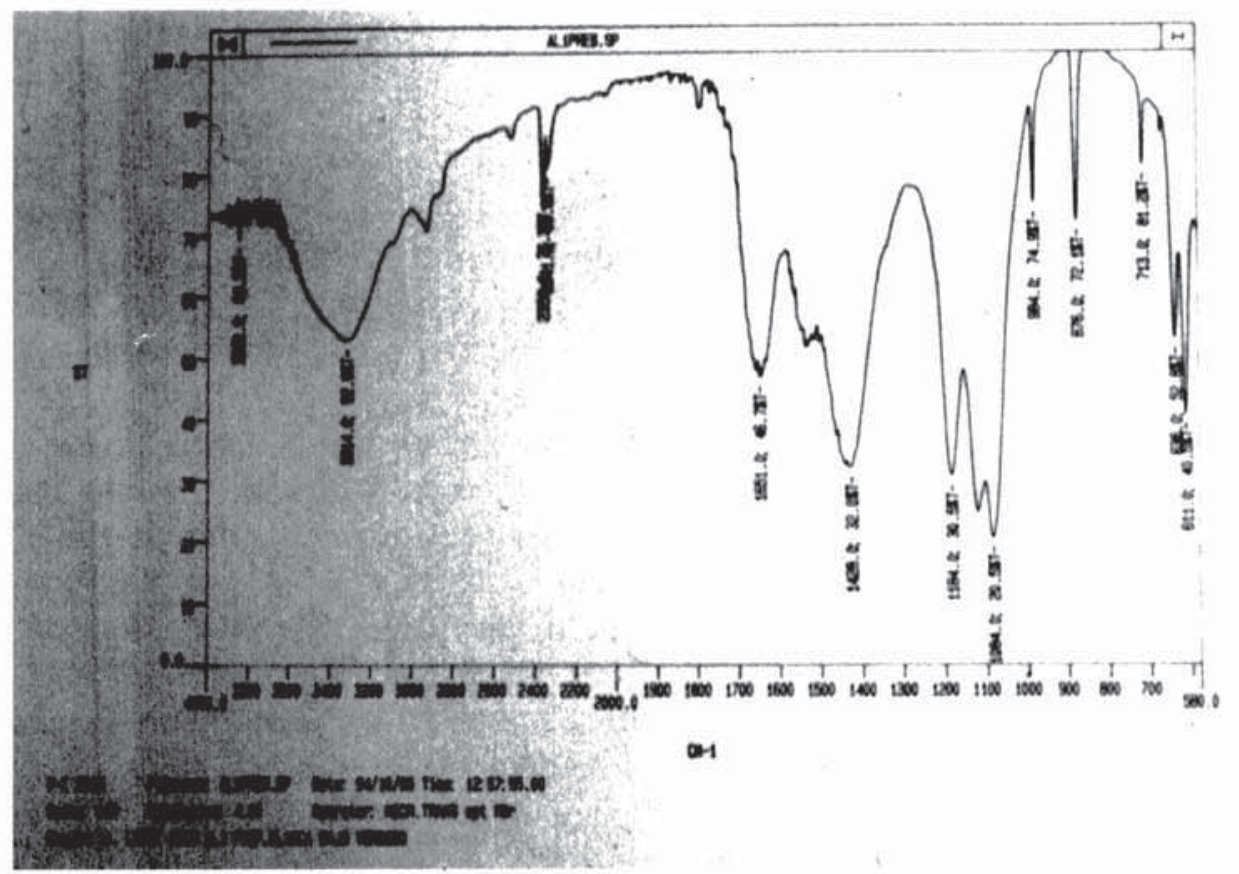

Figura 7. Espectro infrarrojo correspondiente a una muestra de preparación de la obra «El muchacho del tirador..

La cromatografía de gases es una forma especial del procedimiento general cromatográfico de separar una mezcla en sus componentes. La separación se consigue mediante la distribución de los componentes vaporizados entre dos medios de separación. La muestra se introduce en una fase móvil y es transportada a lo largo de la columna que contiene una fase estacionaria distribuida. El reparto entre las dos fases aprovecha las diferencias entre las propiedades físicas y químicas de los componentes de la muestra. Finalmente se obtiene un registro gráfico frente al tiempo.

Se analizan muestras por este método y se obtienen cromatogramas del tipo del de la Fig.8, que corresponde a una muestra de capa pictórica. Las muestras fueron tratadas previamente, para lo cual se realizó una metilación directa, ya que en este caso lo que se están analizando son los ésteres metílicos de los ácidos grasos. En el cromatograma se observa que las señales más importantes corresponden a los tiempos de retención de los ácidos azelaico, palmítico y esteárico. El cálculo de las relaciones de concentración $\mathrm{A} / \mathrm{P}$ y $\mathrm{P} / \mathrm{S}$ nos indican que se trata de un aceite secante, y en concreto de un aceite de lino, ya que si estos valores se comparan en los diagramas de distribución correspondientes para sustancias conocidas, y tratadas 


\section{Métodos de análisis aplicados al estudio de la Obra de Arte}

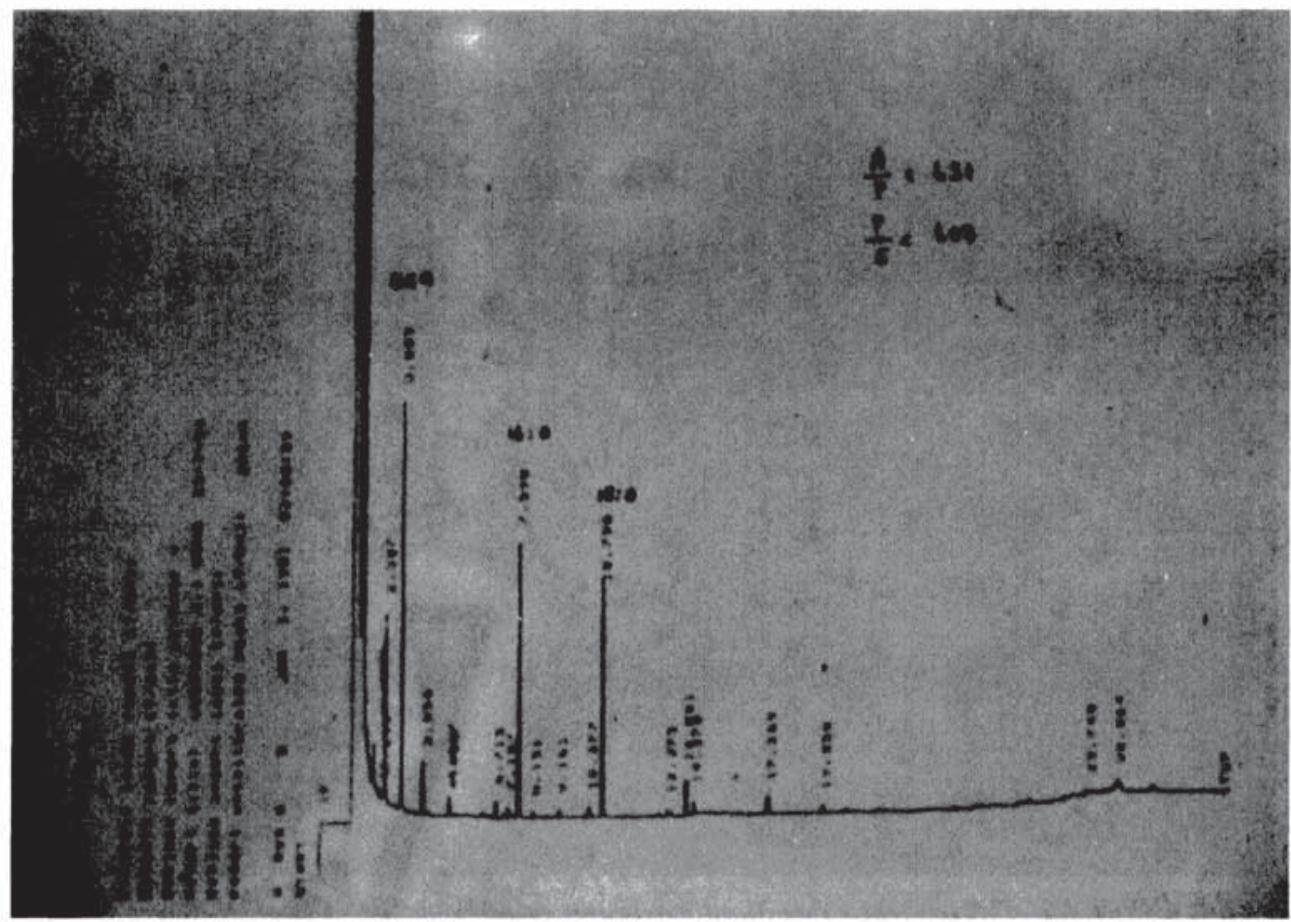

Figura 8. Cromatograma de gases de una muestra de capa pictórica que contiene aceite de lino.

previamente en las mismas condiciones cromatográficas, podemos así deducirlo.

Pero veíamos en el esquema general que dentro de los métodos microscópicos a nuestro alcance, recurríamos también en ciertas ocasiones a la técnica de microscopía electrónica de barrido para caracterizar la superficie de la muestra. El microscopio electrónico enfoca un fino haz de electrones acelerados sobre la superficie de la muestra, que puede ser, en función de su tamaño, un objeto completo o bien muestras previamente tratadas, como las que hemos incluido en una resina para el análisis por microscopía óptica. Este haz de electrones - electrones primarios- que recorre mediante líneas paralelas la muestra sobre la que incide, provoca un desprendimiento de electrones secundarios, junto con radiación $\mathrm{X}, \mathrm{y}$ todos estos pueden ser utilizados, mediante los detectores apropiados, para dar información sobre la superficie de la muestra. Por tanto, nos facilita una imagen de la topografía de la muestra en una pantalla, imagen que a su vez podemos fotografiar.

La circunstancia de que el microscopio electrónico esté acoplado a un detector de rayos $\mathrm{X}$ por dispersión de energía, nos permite un análisis puntual muy preciso de los elementos presentes en la muestra, de mane- 


\section{Carmen Muro García}

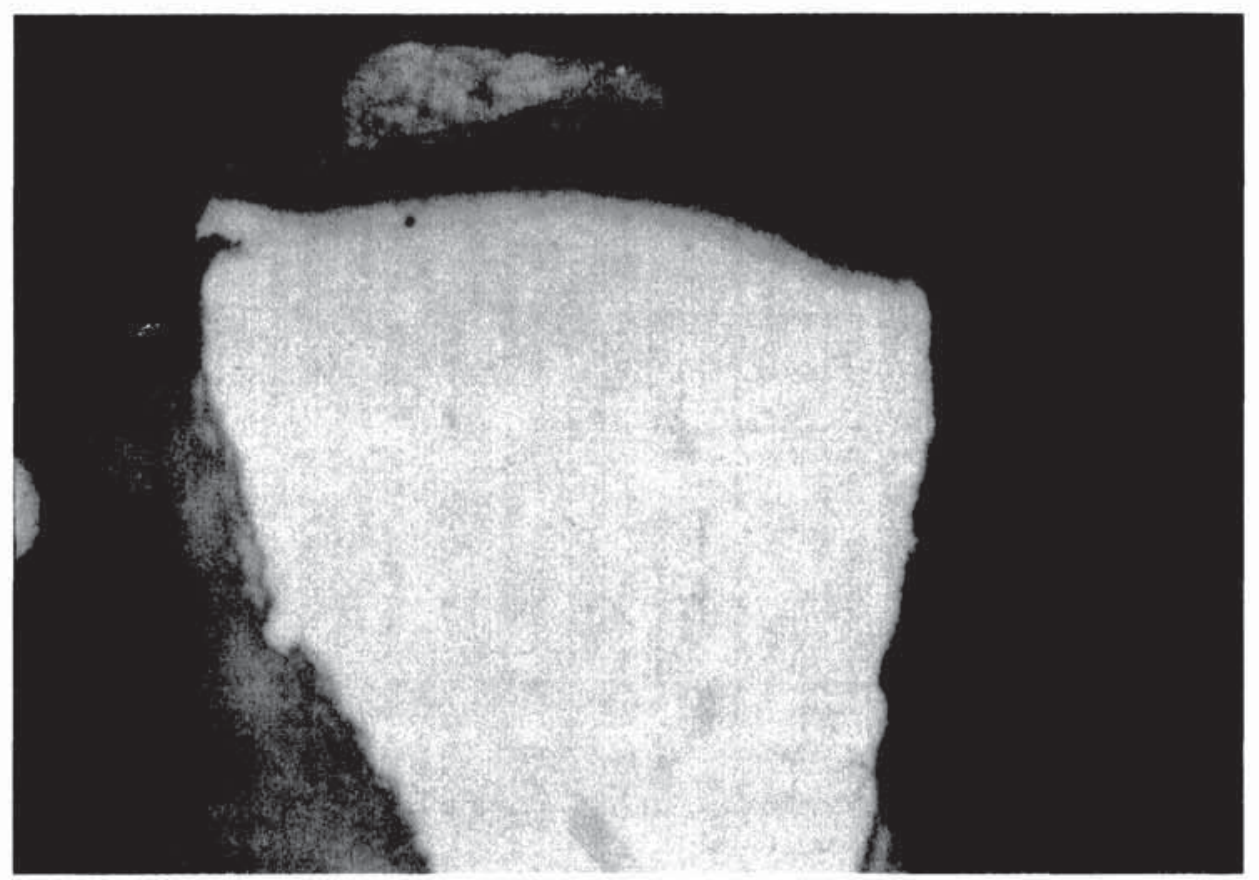

Figura 9. Microfotografía del corte estratigráfico de una muestra violeta de la obra "Negro ascendente " de José Guerrero.

ra que pueden obtenerse mapeados de distribución de elementos en una superficie.

Por ejemplo, del cuadro de José Guerrero titulado "Negro ascendente«, tomamos este ejemplo que nos permite apreciar la diferencia entre los dos violetas presentes en capa pictórica que visulizábamos por microscopía óptica (Fig.9). En la imagen de microscopía electrónica por electrones retrodispersados, se observa en la capa superior un violeta de cobalto $\left[\mathrm{Co}_{3}\left(\mathrm{PO}_{4}\right)_{2}\right]$, y en la capa inferior la mezcla de violeta de cobalto y violeta de manganeso $\left[\left(\mathrm{NH}_{4}\right)_{2} \mathrm{Mn}_{2}\left(\mathrm{P}_{2} \mathrm{O}_{7}\right)_{2}\right]$ (Fig.10).

Hemos repasado brevemente algunos de los diferentes tipos de técnicas que utilizamos con más frecuencia para la caracterización de las obras (pigmentos, soporte, aglutinantes y productos de alteración, así como datos sobre las intervenciones que hayan podido producirse), y cómo estos datos se van complementando, dependiendo naturalmente del objetivo del análisis que se plantee. De esta manera comprobamos que por sí misma en muchos casos una sola técnica no es suficiente y es la complementariedad de los datos aportados la que nos posibilitará la realización del estudio sobre el estado de la obra.

El desarrollo de la actividad científica como método de aproximación al conocimiento de la obra de arte pone de relieve la creciente y 


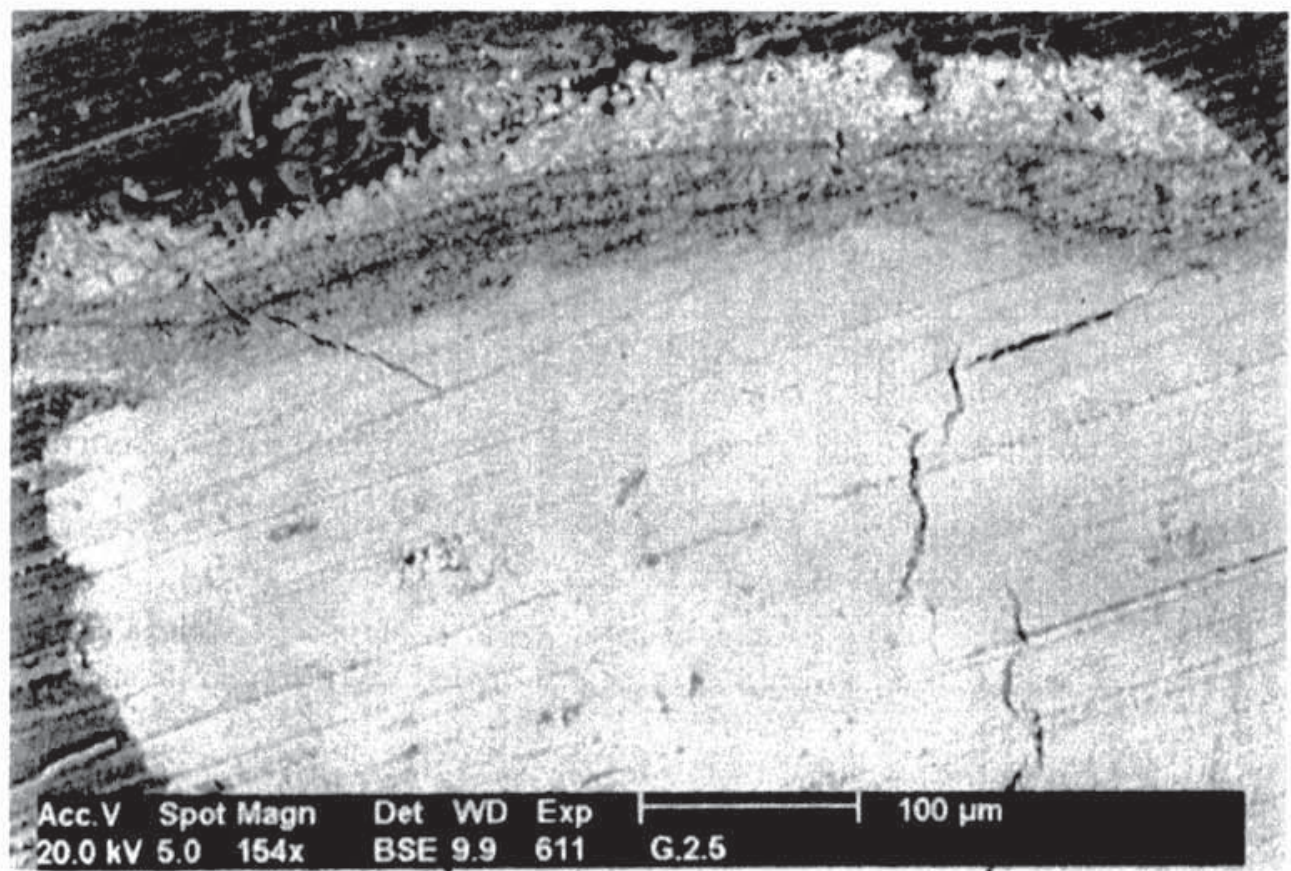

Figura 10. Microscopía electrónica de barrido. Imagen por electrones retrodispersados de la muestra violeta de la Figura 9.

necesaria perspectiva pluridisciplnar en el campo de la conservación del patrimonio cultural. Tradicionalmente el campo de conocimiento de la obra de arte se ha realizado a través de la percepción visual, así como también de toda la información ofrecida por el conocimiento documental, tanto de la época de su creación, como de las sucesivas aportaciones críticas o históricas de la misma. Esta aproximación ha dado como resultado un fecundo campo de interpretación de la obra de arte, de sus circunstancias sociales, relaciones o influencias culturales, contexto histórico, así como sobre las propias cualidades artísticas y creativas que se derivan de la propia contemplación del objeto.

La sucesiva incorporación de disciplinas científicas como la física o química al análisis e interpretación de la obra de arte es un hecho relativamente reciente que abre nuevas perspectivas al conocimiento del patrimonio cultural, en general, y al patrimonio mueble en particular. La clásica visión de las ciencias sociales, como la historia, se complementa así con el creciente conocimiento que sobre la propia estructura material aportan estas investigaciones, coexistiendo y enriqueciendose mutuamente las diferentes aproximaciones. Se abre así un sugestivo y prometedor campo a la colaboración pluridisciplinar. 
No se entienda la pluridisciplinaridad como conjunto de conocimientos especializados en parcelas diferentes de una materia. Al contrario debe concebirse como el trabajo en común sobre un mismo campo de investigación o de intervención (en este caso la obra de arte, el patrimonio cultural), al cual cada profesional aporta el bagaje de conocimientos o técnicas propio de su procedencia disciplinar. Historiadores del arte, restauradores, junto con químicos, físicos, biólogos o geólogos, pueden colaborar en profundizar y ampliar en conjunto en el conocimiento de la obra de arte. No debe olvidarse que ese conocimiento y la consecuente difusión para el enriquecimiento cultural de todos es uno de los objetivos esenciales del patrimonio histórico.

Nota: Los aspectos cromáticos son esenciales en el estudio de la obra pictórica. Por ello, aunque las reproducciones de las imágenes sean en blanco y negro, he considerado conveniente no omitir en el texto las explicaciones alusivas a colores, tanto las referidas a la propia obra como a las incorporadas en su estudio, como son los ensayos microquímicos.

\section{Referencias}

${ }^{1}$ Selim AUGUSTI. Les Méthodes d'analyse appliquées aux neuvres d'art et aux antiquités. IIC. Recent Advences in Conservation. Butterwoths (1963).

${ }^{2}$ Maurice BERNARD. La ciencia al servicio del arte. Museum Internacional (París, UNESCO) $n^{\circ} 183,46 / 3$ (1994), pag. 26 .

${ }^{3}$ Michel VAN DER LAAR, Aviva BURNSTOCK. With paint from Claus\&Fritz: a study of an Amsterdam paintings materials firm (1841-1931). JAIC 36 (1997), 1-16.

${ }^{4}$ Dusan STULIK. Mirar con ojos nuevos. Museum Internacional (París UNESCO) $\mathrm{n}^{\circ} 183,46 / 3$ (1994), pag.21.

GÓMEZ M.L. (1998): La restauración. Examen científico aplicado a la conservación de obras de arte. Cuadernos Arte Cátedra.

MATTEINI, M., MOLES, A. (1996): La Chimica nel Restauro. Nardini Editore.

MILLS, J.S., WHITE, R. (1994): The Organic Chemistry of Museum Objects. Butterworth-Heinemann. 